\title{
Antitumor effects of telomelysin in combination with paclitaxel or cisplatin on head and neck squamous cell carcinoma
}

\author{
NORIO KONDO, MAMORU TSUKUDA, MACHIKO KIMURA, KYOKO FUJITA, ATSUKO SAKAKIBARA, \\ HIDEAKI TAKAHASHI, YUKARI ISHIGURO, GABOR TOTH and HIDEKI MATSUDA \\ Department of Biology and Function in Head and Neck, Yokohama City University \\ Graduate School of Medicine, 3-9 Fukuura, Kanazawa-ku, Yokohama 236-0004, Japan
}

Received July 23, 2009; Accepted October 9, 2009

DOI: $10.3892 /$ or_00000643

\begin{abstract}
Telomelysin (OBP-301) is a telomerase-specific replication-component adenovirus. Telomelysin has a human telomerase reverse transcriptase (hTERT) promoter element which efficiently kills human cancer cells, but not normal cells. The present study investigated the correlation between the antitumor effect of telomelysin and mRNA expression of hTERT and coxsackievirus and adenovirus receptor (CAR) in head and neck squamous cell carcinoma (HNSCC) in vitro and whether telomelysin enhances the antitumor effect of paclitaxel or cisplatin, in vivo using a HNSCC xenograft model. We also determined the optimal order for combining telomelysin treatment and chemotherapy as concurrent treatment, telomelysin treatment first and chemotherapy later, chemotherapy first and telomelysin treatment later for achieving the best anticancer effect. The mRNA expression of hTERT and CAR genes was examined by quantitative RTPCR in 17 HNSCC cell lines. There was no significant correlation between the growth inhibition of telomelysin (ID $_{50}$ for day 3, 5 and 7) in vitro and mRNA expression levels of hTERT and CAR. Regarding the correlation between CAR expression and telomelysin $\mathrm{ID}_{50}$ for day 3 , all cell lines that showed a relative amount of CAR/ B-actin mRNA $>0.4$ had a low telomelysin ID $_{50}$. This may indicate that CAR expression contributes to the efficacy of adenovirus infection and the antitumor activity of telomelysin in early stages of treatment. In our in vivo study, combining telomelysin and paclitaxel had an additive effect regardless of treatment order. On the other hand, combining telomelysin and cisplatin had additive effect only when cisplatin treatment preceded telomelysin treatment. These results suggest that
\end{abstract}

Correspondence to: Dr Norio Kondo, Department of Biology and Function in Head and Neck, Yokohama City University Graduate School of Medicine, 3-9 Fukuura, Kanazawa-ku, Yokohama 236-0004, Japan

E-mail: t076022c@yokohama-cu.ac.jp

Key words: telomelysin, paclitaxel, cisplatin, head and neck squamous cell carcinoma, human telomerase reverse transcriptase, coxsackievirus and adenovirus receptor paclitaxel is considered innocuous for replication of telomelysin, however cisplatin may influence replication of telomelysin.

\section{Introduction}

Head and neck squamous cell carcinoma (HNSCC) is the fifth most common cancer; 500,000-900,000 cases are newly diagnosed annually worldwide (1). The standard treatment for advanced HNSCC includes surgery, radiotherapy and chemotherapy. These treatments modalities prolong the survival of individuals with advanced cases of HNSCC, however the treatment benefit is typically temporary in advanced disease (2). Thus, novel and more effective antitumor agents e.g. targeted molecular therapy, immunotherapy and gene therapy are necessary for treating advanced HNSCC. To improve the therapeutic index, what is needed are agents that target only tumor cells and not normal cells.

Oncolytic adenoviruses have been developed for the treatment of human cancer. These viruses are designed to replicate and selectively kill cancer cells while having a minimum effect on normal cells (3). Such vectors have been approved for clinical trials (4-8). However preclinical and clinical studies have revealed that the clinical application of these agents is hampered by their weak anticancer activity. Therefore, the development of strategies that maximize their anticancer activity is essential to the success of these agents in targeting cancer.

Telomerase is a ribonucleoprotein complex responsible for the complete replication of chromosomal ends (9). Human telomerase reverse transcriptase (hTERT) positively regulates telomerase at the transcriptional level and shows a selectively high activity in growing neoplastic tissues and cells. Many studies have shown that telomerase activity is expressed in $>85 \%$ of human cancers, but only in a few normal somatic cell types $(10,11)$. Therefore, telomerase is an attractive target for the treatment of cancer. Telomelysin (OBP-301) is a telomerase-specific replication-component adenovirus that induces selective E1 expression and exclusively kills human cancer cells (12-15).

Adenoviruses initiate both infection and adenovectormediated gene transfer by attachment of their fiber knobs to a cell surface receptor, the coxsackievirus and adenovirus receptor (CAR) (16). CAR, the primary high-affinity receptor for adenoviruses, is a 46-ku transmembrane glycoprotein and 
belongs to the immunoglobulin superfamily $(17,18)$. Expression of CAR has been studied in numerous cell lines (19-25). In these studies, high expression of CAR correlated with increased adenoviral infection efficacy; cells lacking CAR or expressing low levels of CAR were resistant to adenovirus infection.

These findings led us to examine the correlation between the antitumor effect of telomelysin and mRNA expression levels of hTERT and CAR. Antitumor effect of telomelysin for HNSCC was reported previously (26) and we further examined the combination therapy of telomelysin and chemotherapy (cisplatin or paclitaxel) for the purpose improving the antitumor effect against HNSCC.

\section{Materials and methods}

Adenovirus and chemotherapeutic agents. We used the recombinant replication-selective, tumor-specific adenovirus vector telomelysin (OBP-301), in which the hTERT promoter element drives the expression of E1A and E1B genes linked with an internal ribosome entry site $(12,13)$. Telomelysin was kindly provided by Oncolys Biopharma (Tokyo, Japan). Paclitaxel $\left(\right.$ Taxol $\left.^{\circledR}\right)$ and cisplatin (Briplatin ${ }^{\circledR}$ ) were purchased from Bristol-Myers Squib (Park Avenue, NY). Paclitaxel and cisplatin were diluted with saline just before use for in vivo studies.

Cell lines and culture conditions. Seventeen human HNSCC cell lines were examined in this study. The origins of these cell lines were the oral floor (YCU-OR891), hypopharynx (YCU-H891), mesopharynx (YCU-M862, KCC-M871 and YCU-M911), larynx (KCC-L871 and YCU-L891), tongue (KCC-T871, KCC-T873, YCU-T891, YCU-T892 and HSC3), and maxillary sinus (KCC-MS871 and YCU-MS861) and metastatic tumors from different tongue carcinomas (KCCTCM901, KCC-TCM902 and KCC-TCM903). These cell lines were maintained in RPMI-1640 medium (Life Technologies Inc., Tokyo, Japan) supplemented with $10 \%$ fetal bovine serum (Gibco, Grand Island, NY), 2 mmol/1 Lglutamine, $100 \mathrm{U} / \mathrm{ml}$ penicillin and $100 \mu \mathrm{g} / \mathrm{ml}$ streptomycin. These cells were incubated at $37^{\circ} \mathrm{C}$ in a moist atmosphere containing $5 \% \mathrm{CO}_{2}$.

Detection of hTERT and CAR mRNAs by quantitative RT-PCR. Cells were grown until nearly confluent in RPMI-1640 supplemented with $10 \%$ FBS. Total RNA extracted from 17 human HNSCC cells with phenol solution (Isogen; Nippon Gene Inc., Tokyo, Japan) according to the manufacturer's protocol. One microgram of total RNA was converted into cDNA by using Takara RNA PCR kit (AMV) Ver.3.0 (Takara Bio Inc., Tokyo, Japan). The quantification of relative expression levels of $\beta$-actin and hTERT were carried out with Platinum Quantitative PCR SuperMix-UDG with ROX (Invitrogen, Carlsbad, CA) using an ABI Prism ${ }^{\circledR} 7500$ Sequence Detection System (Applied Biosystems, Foster City, CA). The quantification of relative expression levels of CAR were carried out with TaqMan Gene Expression Master Mix (Applied Biosystems) using an ABI Prism ${ }^{\circledR} 7500$ Sequence Detection System (Applied Biosystems). PCR was conducted using the following cycle parameters: $50^{\circ} \mathrm{C}$ for $2 \mathrm{~min}, 95^{\circ} \mathrm{C}$ for $10 \mathrm{~min}$, followed by 60 cycles at $95^{\circ} \mathrm{C}$ for $15 \mathrm{sec}$ and $60^{\circ} \mathrm{C}$ $1 \mathrm{~min}$. The primers used to detect each factor were as follows: ß-actin: HLUX3000126 (Invitrogen); hTERT: HLUX3011813 (Invitrogen); CAR: Hs00154661 (Applied Biosystems). The results were normalized by the corresponding B-actin levels. Each PCR amplification was performed at least three times and the mean was calculated.

In vivo antitumor activity studies. Female BALB/c nu/nu nude mice, 6-week-old, were obtained from Oriental Yeast (Tokyo, Japan). The mice were maintained in a laminar flow room with a constant temperature and humidity. The animals were maintained and experiments were conducted at the Yokohama City University School of Medicine, Laboratory Animal Facility. The experiments were conducted according to the guidelines for animal experiments set by the Animal Experiment Committee of Yokohama City University School of Medicine. Suspensions of YCU-H891 cells (100 $\mu 1$ ) (final concentration, $1 \times 10^{7}$ cells $/ 100 \mu \mathrm{l}$ ) were injected s.c. into the right flank of the mice. Tumor-bearing mice were randomized $(\mathrm{n}=6)$ when the mean tumor volume was $50-100 \mathrm{~mm}^{3}$. Each group was closely matched before treatment, which began one week after cell transplantation. The mice were treated i.t. with telomelysin $\left(5 \times 10^{7} \mathrm{PFU} / \mathrm{mouse}\right)$ or PBS (vehicle) on day 7 or 14. Paclitaxel or saline (vehicle) was administered i.p. $(7.5 \mathrm{mg} / \mathrm{kg} /$ day 2 times on day 7 and 11 or day 14 and 18). Cisplatin or saline (vehicle) was administered i.p. (2.2 mg/ $\mathrm{kg}$ /day on day 7 or 14 ). The control mice were not treated with any material. Treatment group number was 15 and the combination of treatment and administration orders are shown in Fig. 3A and B. Tumor diameters in the control and treated groups were measured weekly with a Vernier caliper. Tumor volume $(\mathrm{V})$ was determined by the equation: $\mathrm{V}=\mathrm{ab}^{2} / 2$ ( $\mathrm{a}=$ length; $\mathrm{b}=$ width).

Evaluation of apoptosis. Terminal deoxynucleotidyl transferase-mediated cUDP nick end-labeling (TUNEL) was done for the evaluation of apoptosis. This was evaluated using an Apoptosis In Situ Detection Kit (Wako Chemical, Osaka, Japan). The apoptotic index was calculated as the percentage of positive cell nuclei stained with peroxidase relative to the total number of cells in the fields at x400 magnification. Six fields per histological section were included in the analysis.

Evaluation of vessel density. In order to estimate the vessel density, the vessels in the tumor tissues were stained with an anti-CD31 antibody (1:200; Dako, Denmark). The tumors were excised and blocked with $2 \%$ goat serum and $1 \%$ bovine serum albumin in PBS and stained with the antibody. Slides were developed using 3,3'-diaminobenzidine substrate biotinylated peroxidase reagent (Vector Laboratories, Inc., Burlingame, CA). Vessel density was determined by counting the stained vascular endothelial cells in the fields at $\times 100$ magnification. Six fields per histological section were included in the analysis.

Statistical analysis. For statistical analyses of the correlation between $\mathrm{ID}_{50}$ of telomelysin and mRNA expression of hTERT or CAR, we used Spearman's correlation coefficient or by the rank test. For statistical analyses of in vivo antitumor activity, 


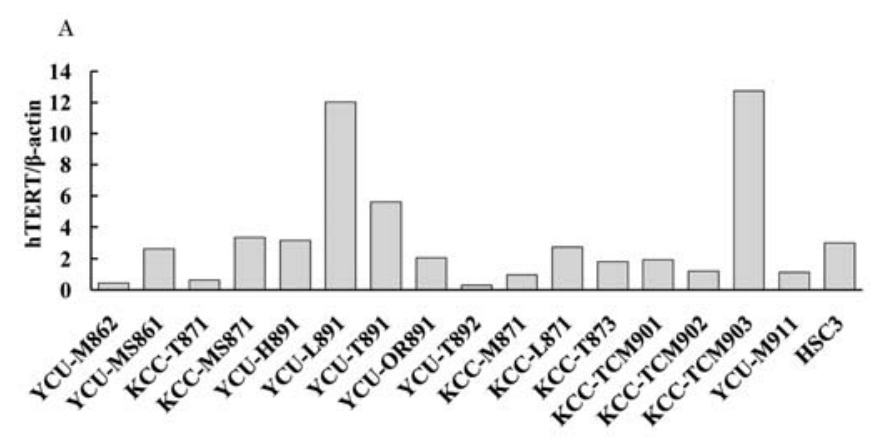

B

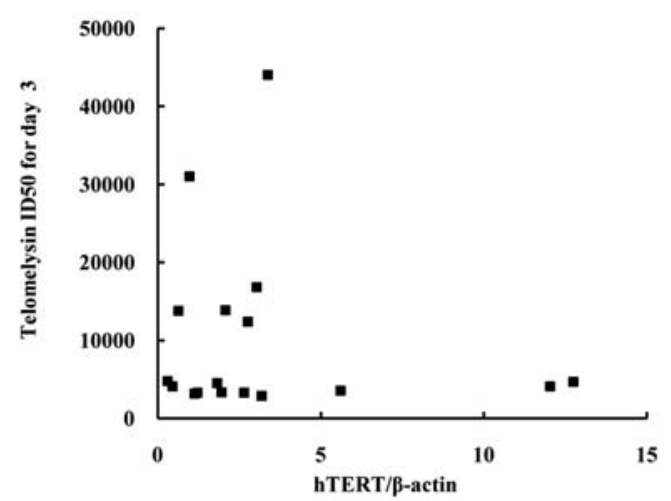

$\mathrm{C}$

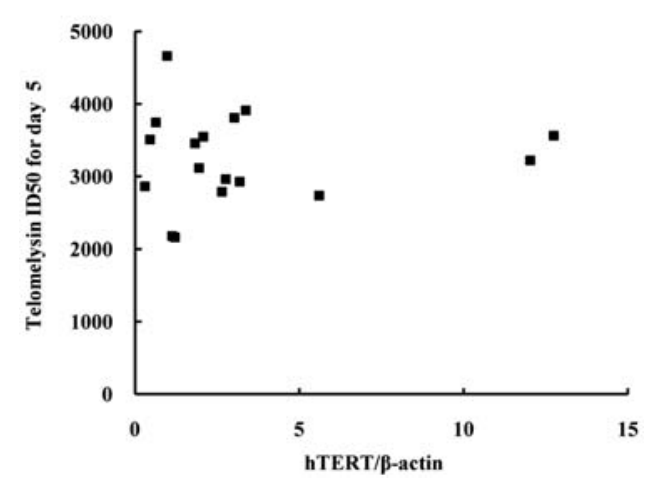

D

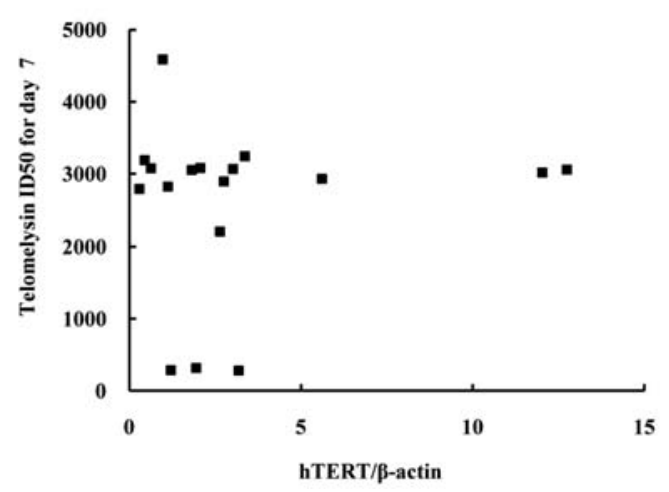

Figure 1. (A) Expression of hTERT mRNAs in HNSCC cell lines. Expression of mRNA was determined by real-time RT-PCR in 17 HNSCC cell lines. The level of each mRNA was normalized relative to $B$-actin mRNA. (B) Correlation between hTERT expression and telomelysin $\mathrm{ID}_{50}$ for day 3 . (C) Correlation between hTERT expression and telomelysin $\mathrm{ID}_{50}$ for day 5. (D) Correlation between hTERT expression and telomelysin $\mathrm{ID}_{50}$ for day 7 . Correlation was not observed in all groups $(\mathrm{P}<0.05)$. Statistical correlation was analyzed with Speaman's correlation coefficient or by the rank test.

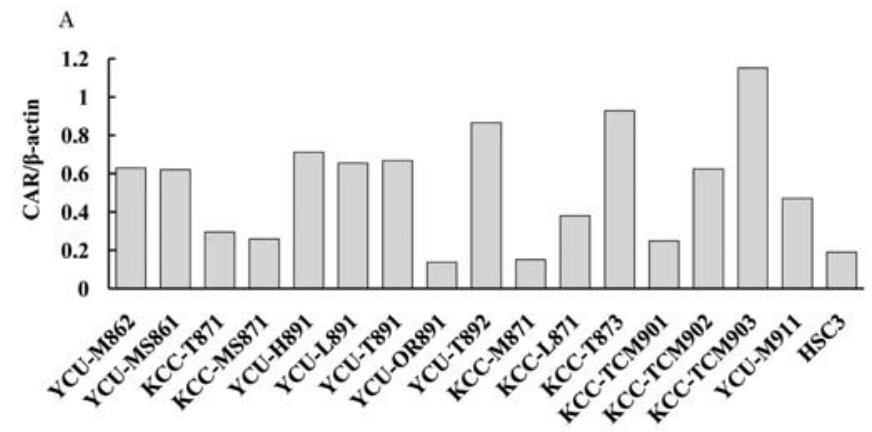

B

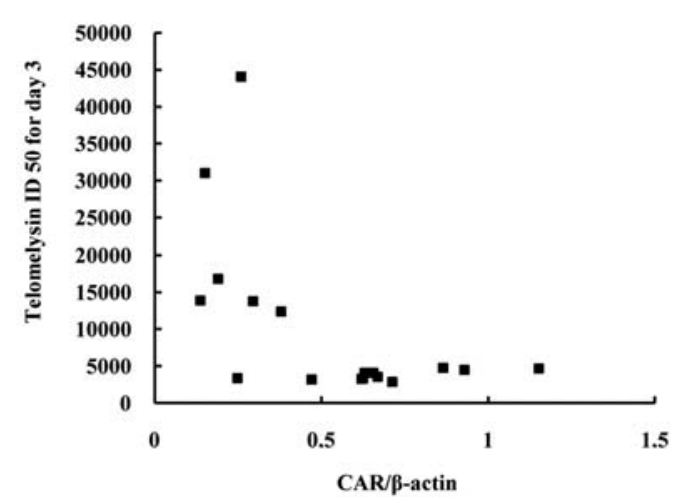

C

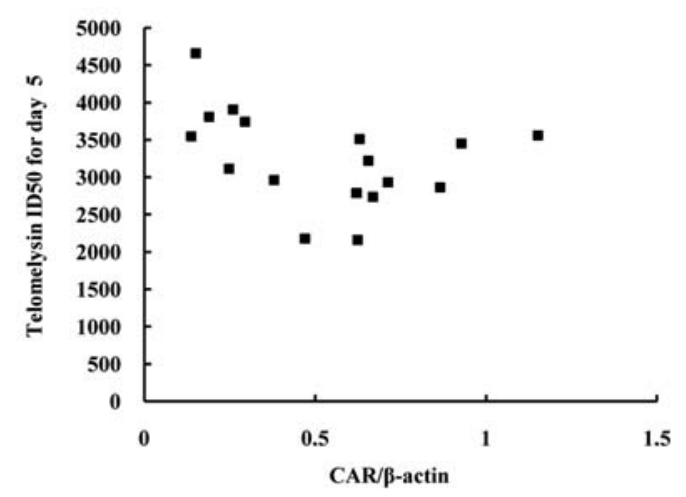

D

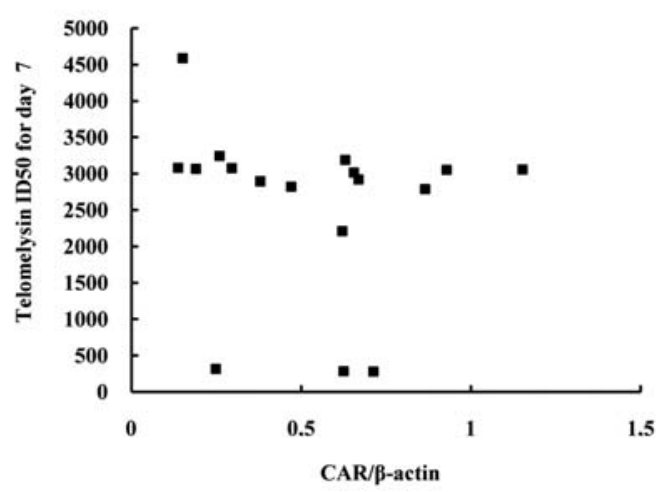

Figure 2. (A) Expression of CAR mRNAs in HNSCC cell lines. Expression of mRNA was determined by real-time RT-PCR in 17 HNSCC cell lines. The level of each mRNA was normalized relative to B-actin mRNA. (B) Correlation between CAR expression and telomelysin $\mathrm{ID}_{50}$ for day 3. (C) Correlation between CAR expression and telomelysin $\mathrm{ID}_{50}$ for day 5. (D) Correlation between CAR expression and telomelysin $\mathrm{ID}_{50}$ for day 7 . Correlation was not observed in all groups $(\mathrm{P}<0.05)$. Statistical correlation was analyzed with Speaman's correlation coefficient or by the rank test. 


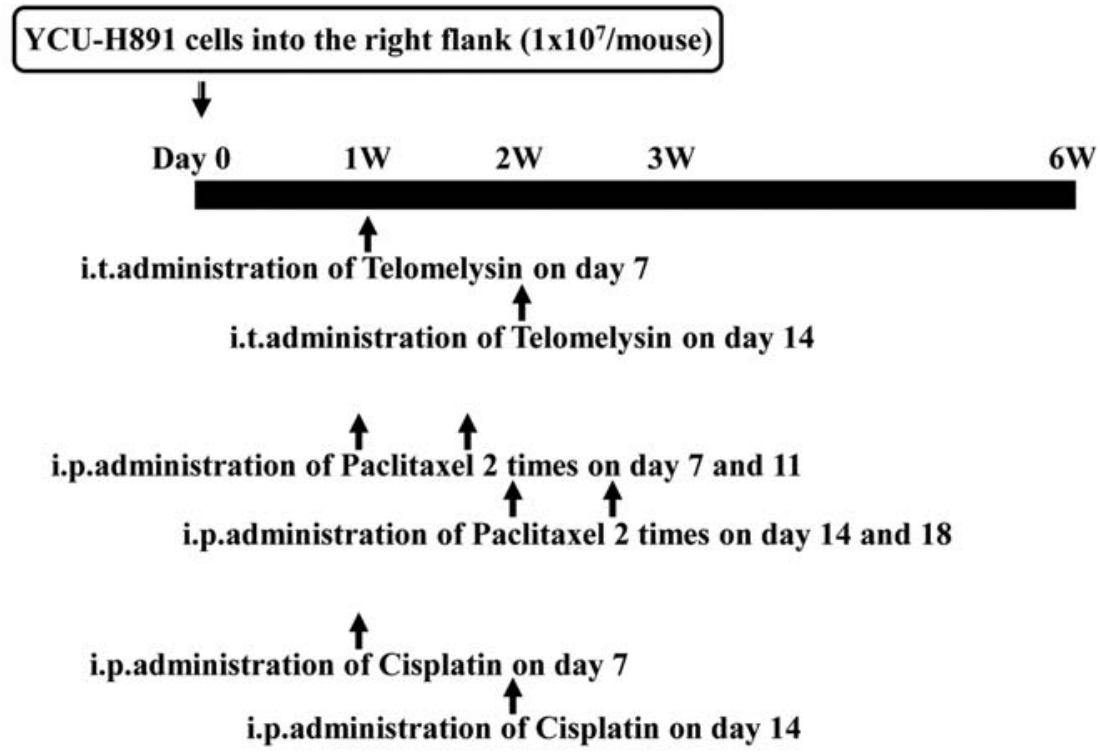

B

\begin{tabular}{|c|l|l|}
\hline Group & The number of mice & \multicolumn{1}{|c|}{ Treatment } \\
\hline 1 & 6 & Control \\
\hline 2 & 6 & PBS i.t. $\rightarrow$ Saline i.p. \\
\hline 3 & 6 & Saline i.p. $\rightarrow$ PBS i.t. \\
\hline 4 & 6 & Paclitaxel $7.5 \mathrm{mg} / \mathrm{kg}$ i.p. $\rightarrow$ PBS i.t. \\
\hline 5 & 6 & PBS i.t. $\rightarrow$ Paclitaxel $7.5 \mathrm{mg} / \mathrm{kg}$ i.p. \\
\hline 6 & 6 & Telomelysin $5 \times 10^{7}$ PFU/body i.t. $\rightarrow$ Saline i.p. \\
\hline 7 & 6 & Saline i.p. $\rightarrow$ Telomelysin $5 \times 10^{7}$ PFU/body i.t. \\
\hline 8 & 6 & Telomelysin $5 \times 10^{7}$ PFU/body i.t. $\rightarrow$ Paclitaxel $7.5 \mathrm{mg} / \mathrm{kg}$ i.p. \\
\hline 9 & 6 & Paclitaxel $7.5 \mathrm{mg} / \mathrm{kg}$ i.p. $\rightarrow$ Telomelysin $5 \times 10^{7}$ PFU/body i.t. \\
\hline 10 & 6 & Telomelysin $5 \times 10^{7}$ PFU/body i.t., Paclitaxel $7.5 \mathrm{mg} / \mathrm{kg}$ i.p. concurrent \\
\hline 11 & 6 & Cisplatin $2.2 \mathrm{mg} / \mathrm{kg}$ i.p. $\rightarrow$ PBS i.t. \\
\hline 12 & 6 & PBS i.t. $\rightarrow$ Cisplatin $2.2 \mathrm{mg} / \mathrm{kg}$ i.p. \\
\hline 13 & 6 & Telomelysin $5 \times 10^{7}$ PFU/body i.t. $\rightarrow$ Cisplatin $2.2 \mathrm{mg} / \mathrm{kg}$ i.p. \\
\hline 14 & 6 Cisplatin $2.2 \mathrm{mg} / \mathrm{kg}$ i.p. $\rightarrow$ Telomelysin $5 \times 10^{7}$ PFU/body i.t. \\
\hline
\end{tabular}

Figure 3. Animal experiment regimens. (A) Administration schedule of telomelysin and chemotherapeutic agents. Mice were inoculated with $1 \times 10^{7}$ YCU-H891 cells on day 0 . Telomelysin $\left(5 \times 10^{7}\right.$ PFU/mouse) was administered i.t. on day 8 or 14 . Paclitaxel $(7.5 \mathrm{mg} / \mathrm{kg}) \mathrm{was}$ administered i.p. 2 times on day 7 and 11 , or day 14 and 18. Cisplatin $(2.2 \mathrm{mg} / \mathrm{kg})$ was administered i.p. on day 7 or 14. (B) The group number was 15 and 6 mice were used in each group. Telomelysin treatment alone and three orders of combination treatment of telomelysin and chemotherapy - telomelysin and chemotherapy concurrently, telomelysin and then chemotherapy, chemotherapy and then telomelysin were examined.

apoptotic index, vessel density, we used Student's paired t-test. A value of $\mathrm{P}<0.05$ was considered significant.

\section{Results}

Effect of expression of hTERT and CAR mRNAs on 17 HNSCC cell lines and correlation with telomelysin $I D_{50}$. To determine the correlation between the telomelysin $\mathrm{ID}_{50}$ and expression of hTERT and CAR, we analyzed levels of mRNA for hTERT and CAR in 17 HNSCC cell lines by quantitative RT-PCR. Although expression levels of hTERT mRNA, which plays a key role in telomerase activation (27), varied widely, all cell lines expressed mRNA encoding hTERT (Fig. 1A). In an earlier study, we restablished the telomelysin $\mathrm{ID}_{50}$ for of day 3, 5 and 7 (26). The correlation between expression levels of hTERT mRNA and telomelysin ID $_{50}$ was not 
significant by Spearman's correlation coefficient or by the rank test (Fig. 1B-D). These results suggest that the hTERT promoter element is an important target in human cancer therapy, but its expression does not correlate with the therapeutic efficacy of telomelysin. Although all cell lines expressed CAR mRNA (Fig. 2A), correlation was not observed between CAR mRNA and the telomelysin $\operatorname{ID}_{50}$ (Fig. 2B-D). The cell lines that showed a relative mRNA value $>0.4$ for the ratio $\mathrm{CAR} / \mathrm{B}$-actin, had a low telomelysin $\mathrm{ID}_{50}$.

In vivo studies of the antitumor effects of telomelysin in combination with cisplatin and paclitaxel on HNSCC xenografts. We reported an inhibitory effect of telomelysin on tumor growth in the HNSCC xenograft model (26). Here we investigated the in vivo antitumor activity of combination therapy of telomelysin and paclitaxel or cisplatin in nude mice bearing YCU-H891 squamous cell carcinoma xenografts. To investigate the influence of chemotherapeutic agents on infection efficacy and antitumor activity combined with telomelysin, we administered combination therapy of telomelysin and chemotherapy in three orders: telomelysin treatment and chemotherapy concurrently, telomelysin treatment and then chemotherapy, chemotherapy and then telomelysin. As shown in Fig. 4A, paclitaxel treatment alone $(7.5 \mathrm{mg} / \mathrm{kg} /$ day, 2 times $)$ or telomelysin alone $\left(5 \times 10^{7} \mathrm{PFU} /\right.$ mouse) did not cause a complete regression of tumors, however combination therapy of the two reduced the tumor volume compared to telomelysin or paclitaxel alone (Fig. 4A). There was a marked reduction in tumor volume after a regimen of concurrent telomelysin and paclitaxel and after paclitaxel then telomelysin compared to telomelysin alone ( $\mathrm{P}<0.05$; Fig. 4A).

Regarding cisplatin, cisplatin then telomelysin caused marked reduction in tumor volume compared to telomelysin alone $(\mathrm{P}<0.05$; Fig. 4B). Combining these two drugs did not show a combination effect on tumor volume for telomelysin then cisplatin or for concurrent telomelysin and cisplatin.

Effect of combination treatment on apoptosis in the xenograft model. Histopathological analysis of the xenograft samples was done to examine apoptotic events, one of antitumor mechanisms (Fig. 5A). The all combination protocols of telomelysin and paclitaxel showed an increased number of TUNEL-positive cells (Fig. 5B). The apoptotic index was significantly higher in tumors from mice treated with telomelysin and paclitaxel concurrently, or with telomelysin then paclitaxel compared to paclitaxel alone $(\mathrm{P}<0.05$; Fig. $5 \mathrm{~B})$.

Telomelysin in combination with cisplatin, for all combination protocols show a higher apoptotic index, but statistical significance was not reached (Fig. 5C).

Effect of combination treatment on microvascular content in the xenograft model. To determine the effects of the combination treatment of telomelysin and paclitaxel or cisplatin on tumor neovascularization, the vessel density of the xenograft tumors was examined to clarify antitumor mechanisms (Fig. 6A). The all combination protocols of telomelysin and paclitaxel showed a lower number of microvessels positive for CD31 staining compared to telomelysin or paclitaxel alone, but there was no statistical significance (Fig. 6B). For the combination of telomelysin and cisplatin, all the protocols

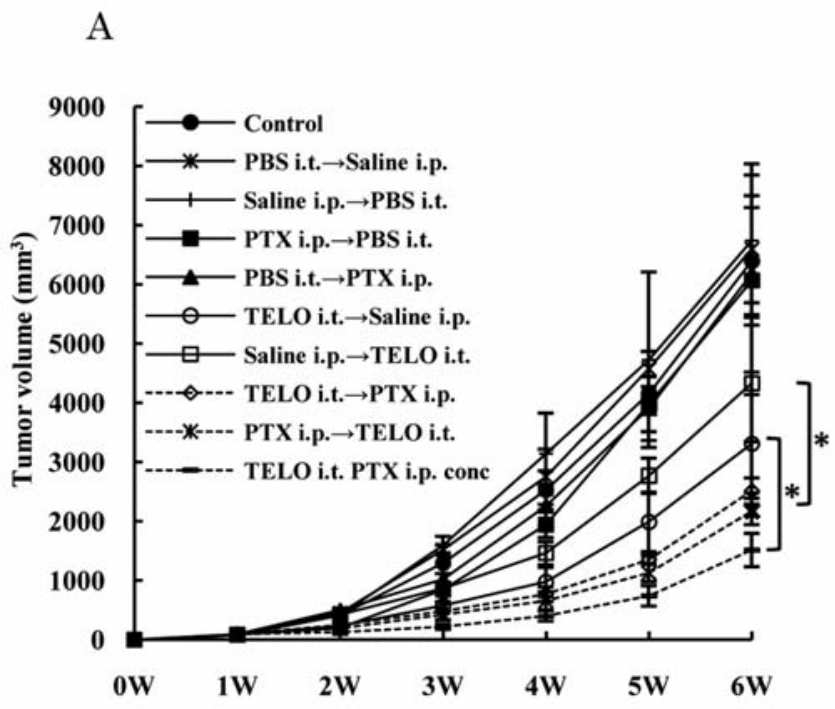

B

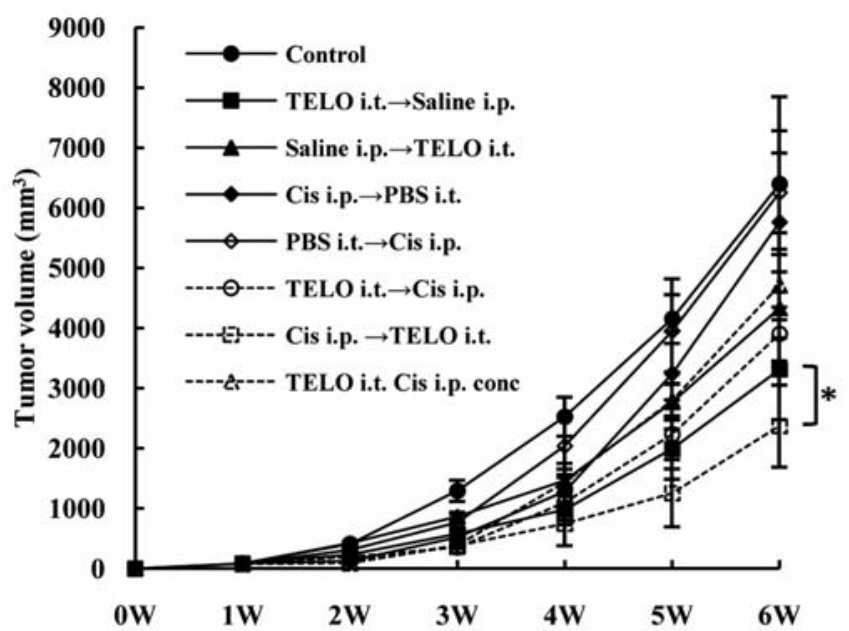

Figure 4. Effect of telomelysin and chemotherapy on the growth of HNSCC xenograft model. (A) The group of combination treatment of telomelysin and paclitaxel concurrently (telomelysin:5x $10^{7} \mathrm{PFU} /$ body i.t. on day 7 , paclitaxel:7.5 mg/kg i.p. 2 times on day 7 and 11) and the group of combination treatment of telomelysin and then paclitaxel (paclitaxel:7.5 mg/kg i.p. 2 times on day 7 and 11, telomelysin:5x10 7 PFU/body i.t. on day 14) demonstrated significant growth inhibition compared to telomelysin alone (telomelysin:5x $10^{7} \mathrm{PFU} /$ body i.t. on day 7 or 14$)\left({ }^{*} \mathrm{P}<0.05\right)$. (B) The group of combination treatment of cisplatin and then telomelysin (telomelysin: $5 \times 10^{7} \mathrm{PFU} /$ body i.t. on day 14 , cisplatin: $2.2 \mathrm{mg} / \mathrm{kg}$ i.p. on day 7 ) demonstrated significant growth inhibition compared to telomelysin alone (telomelysin: $5 \times 10^{7} \mathrm{PFU} /$ body i.t. on day 14$)\left({ }^{*} \mathrm{P}<0.05\right)$. Error bars indicates SE. Significant difference was analyzed by Student's paired t-test.

resulted in almost the same number of microvessels positive for CD31 staining compared to the control group (Fig. 6C).

\section{Discussion}

Telomelysin has been clarified to be effective against human cancers $(12,13,15)$. We also reported that telomelysin has a strong antitumor effect against human HNSCC cell lines in vitro and against a murine HNSCC cell line in vitro and in vivo (26). Telomelysin has a human telomerase reverse 
A

TUNEL $(\times 400)$

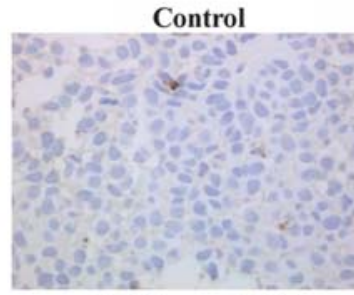

PTX i.p. $\rightarrow$ PBS i.t.

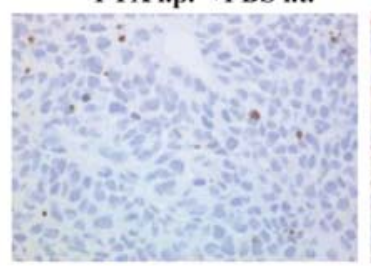

PBS i.t. $\rightarrow$ PTX i.p.

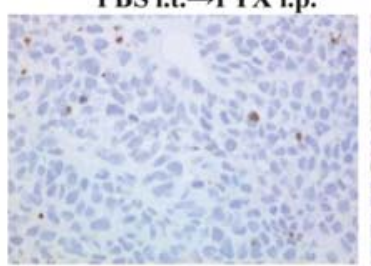

TELO i.t. $\rightarrow$ Saline i.p.

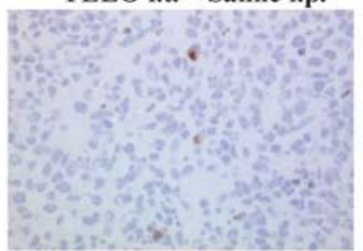

B

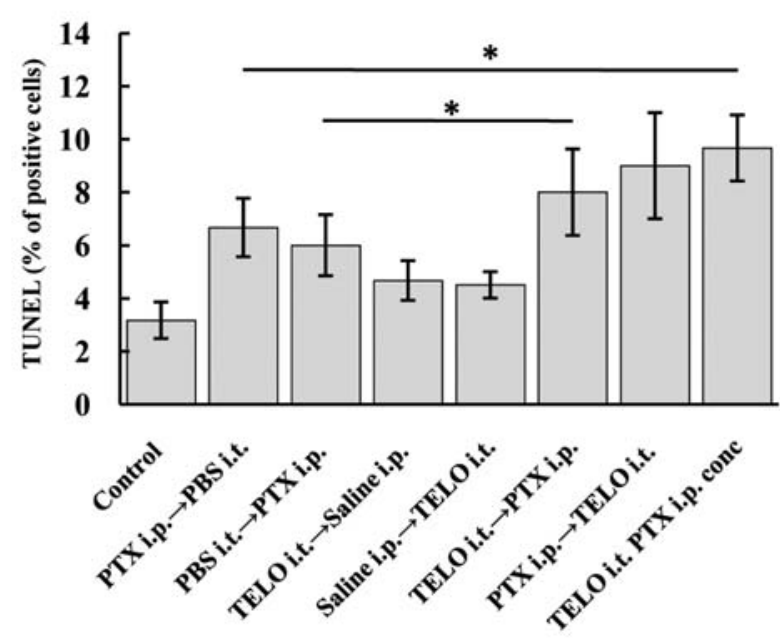

Saline i.p. $\rightarrow$ TELO i.t.

TELO i.t. $\rightarrow$ PTX i.p.

PTX i.p. $\rightarrow$ TELO i.t.

TELO i.t., PTX i.p. cone
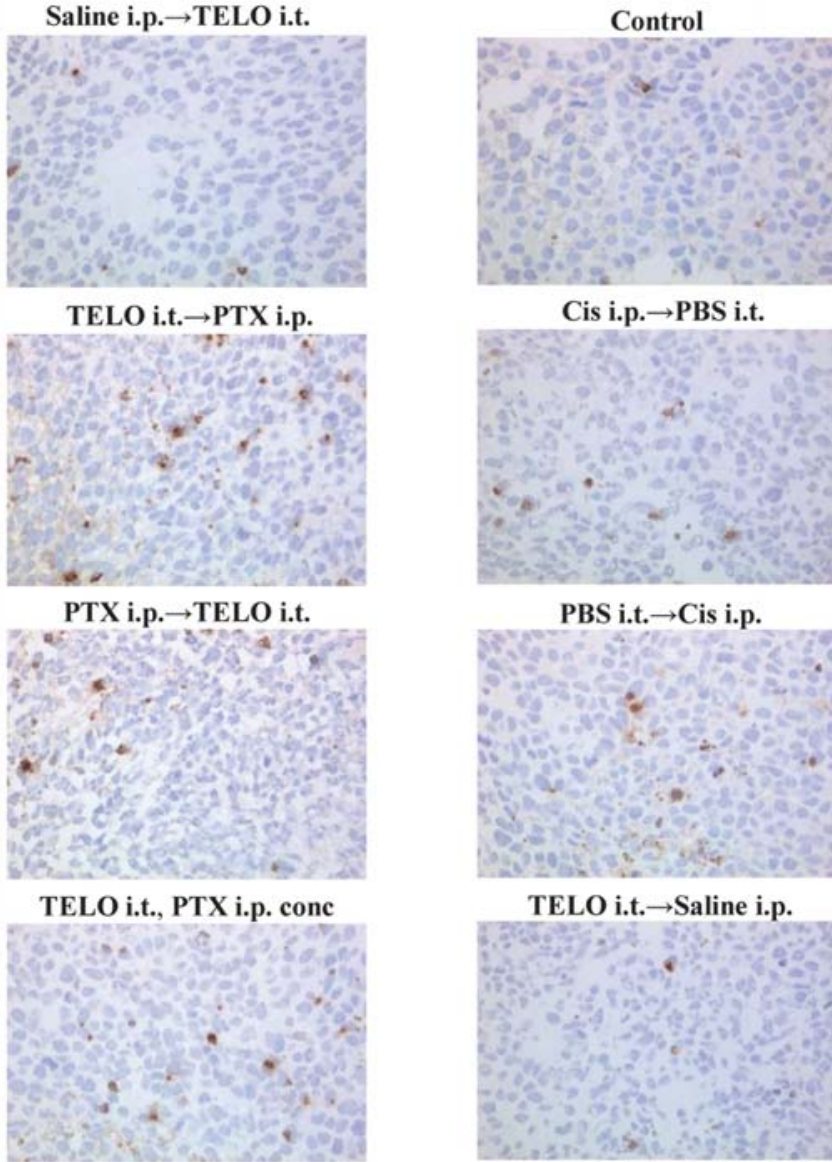

Cis i.p. $\rightarrow$ PBS i.t.

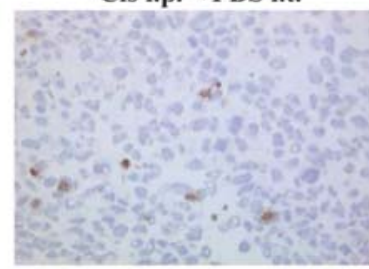

PBS i.t. $\rightarrow$ Cis i.p.

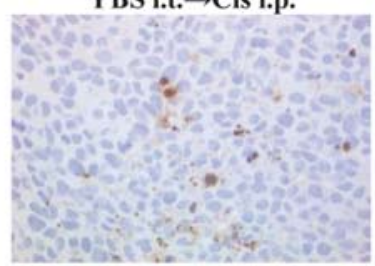

TELO i.t. $\rightarrow$ Saline i.p.

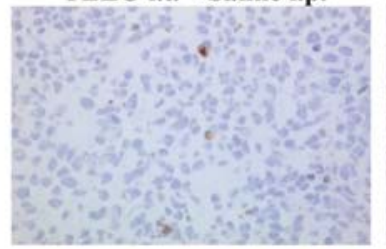

C
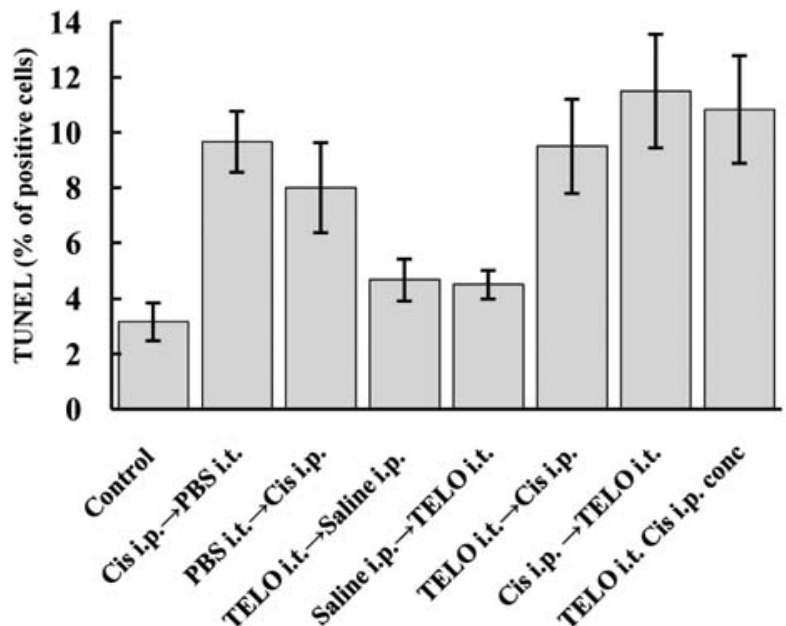

Figure 5. Apoptotic events in YCU-H891 tumor xenografts. Terminal deoxynucleotidyl transferase-mediated cUDP nick end-labeling (TUNEL) staining was utilized. (A) Histopathological analysis of apoptosis by TUNEL about the group of combination treatment of telomelysin and paclitaxel or cisplatin (x400). (B) Effects of telomelysin and paclitaxel on apoptotic index (TUNEL). Statistical significance was observed in the group of telomelysin and paclitaxel concurrently and the group of telomelysin and then paclitaxel compared to paclitaxel alone ( $\left.{ }^{*} \mathrm{P}<0.05\right)$. (C) Effects of telomelysin and cisplatin on apoptotic index (TUNEL). Statistical significance was not observed in the group of combination treatment of telomelysin and cisplatin compared to cisplatin alone $(\mathrm{P}<0.05)$. Data represent mean values $( \pm \mathrm{SD})$. Significant difference was analyzed by Student's paired t-test.

transcriptase promoter that regulates viral replication and efficiently kills human cancer cells. Human telomerase reverse transcriptase (hTERT) positively regulates telomerase at the transcriptional level and shows a selectively high inhibitory activity against growing neoplastic tissues and cells. Therefore, we investigated the relative mRNA expression of hTERT in 17 HNSCC cell lines and evaluated the correlation of these mRNA species with the telomelysin $\mathrm{ID}_{50}$ reported by us 
A

\section{CD31 ( × 100)}

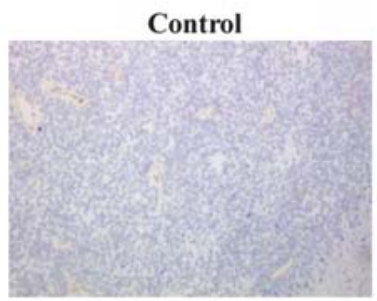

PTX i.p. $\rightarrow$ PBS i.t.

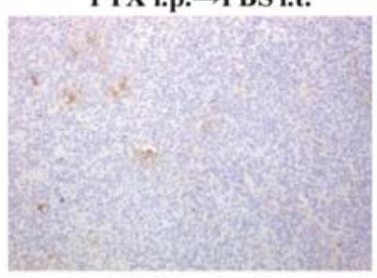

PBS i.t. $\rightarrow$ PTX i.p.

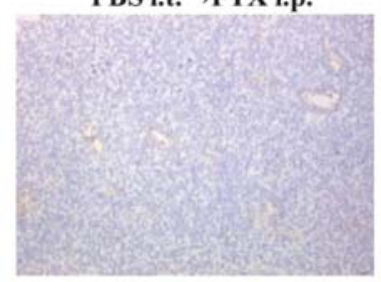

TELO i.t. $\rightarrow$ Saline i.p.

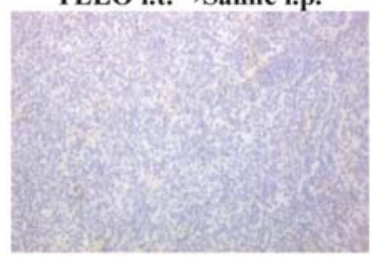

B

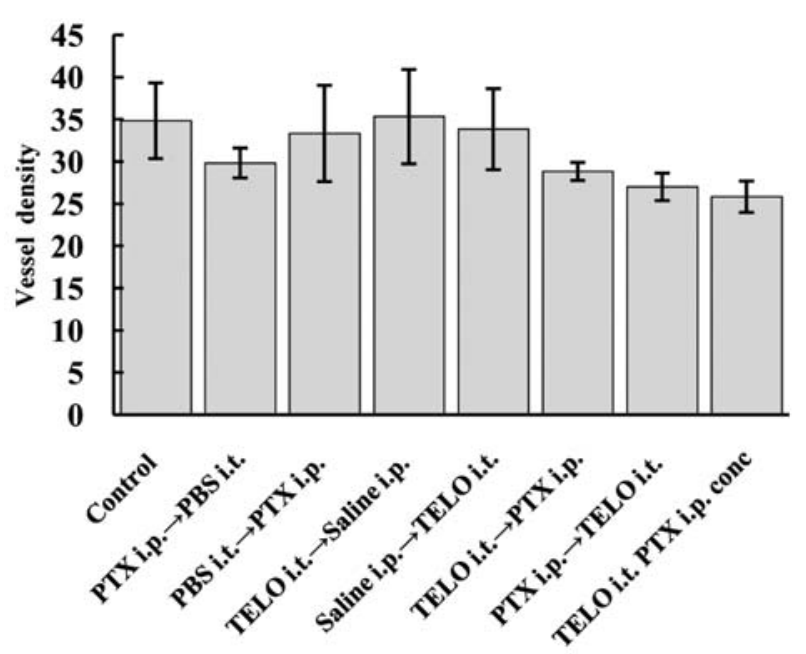

Saline i.p. $\rightarrow$ TELO i.t.

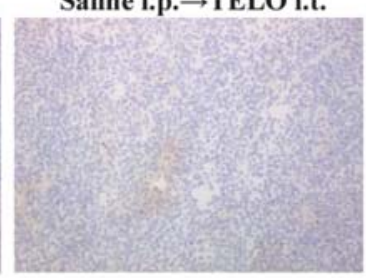

TELO i.t. $\rightarrow$ PTX i.p.

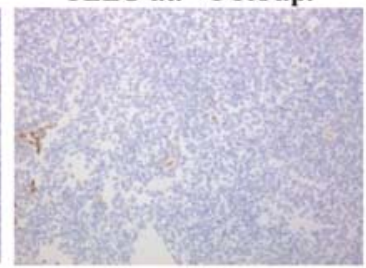

PTX i.p. $\rightarrow$ TELO i.t.

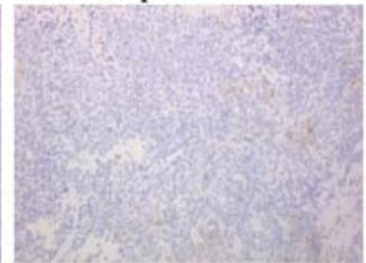

TELO i.t., PTX i.p. cone
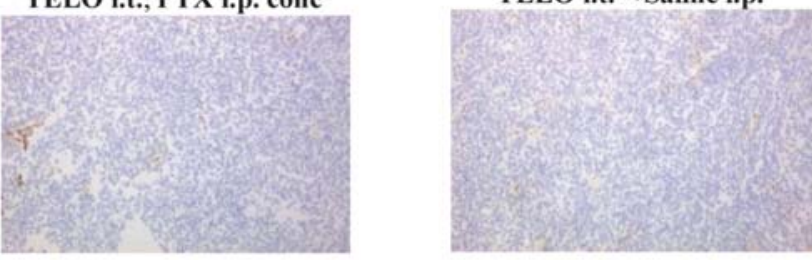

C
TELO i.t. $\rightarrow$ Saline i.p.

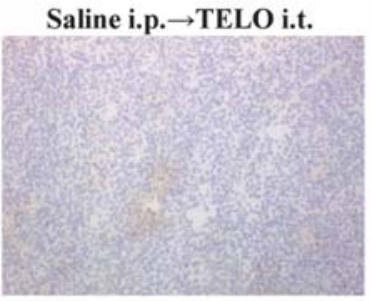

TELO i.t. $\rightarrow$ Cis i.p.

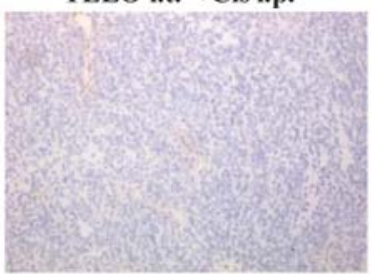

Cis i.p. $\rightarrow$ TELO i.t.
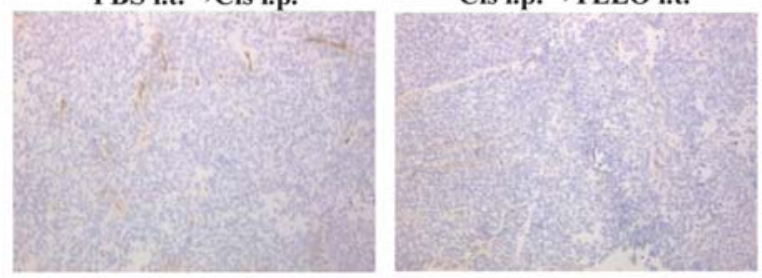

TELO i.t., Cis i.p. conc
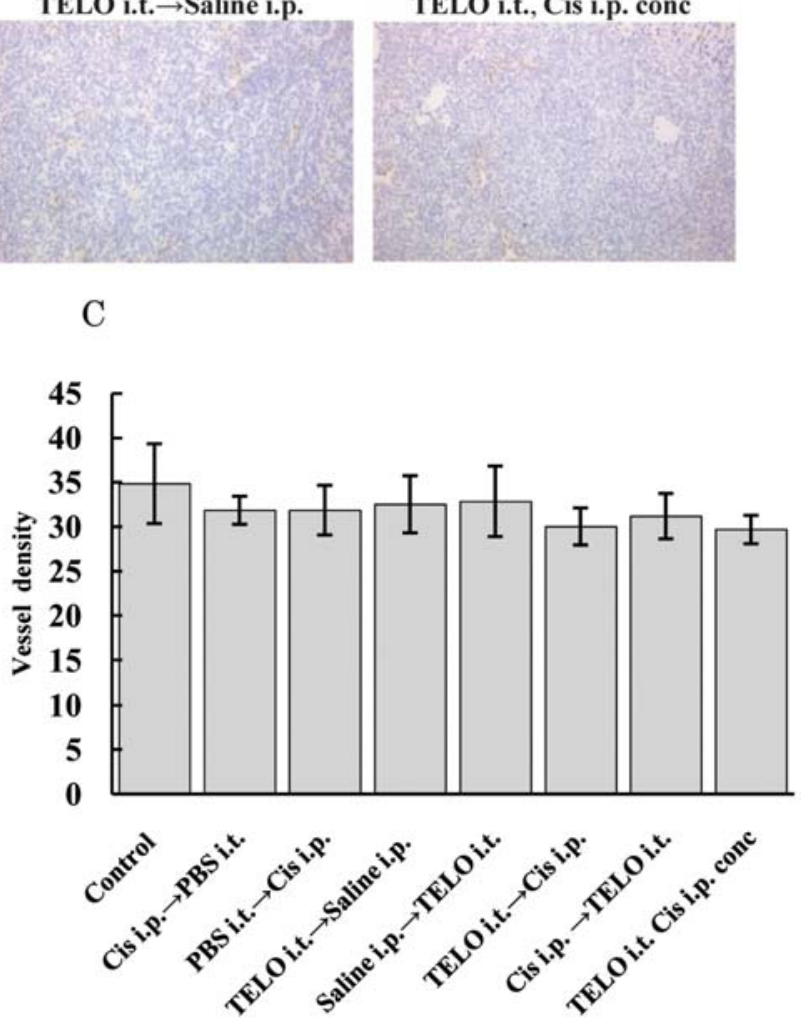

Figure 6. Vessel density in YCU-H891 tumor xenografts. The vessels of tumors were stained with anti-CD31 antibody (x100). (A) Vascular attaining about the group of combination treatment of telomelysin and paclitaxel or cisplatin. (B) Effects of telomelysin and paclitaxel on vessel density. Statistical significance was not observed in the group of combination treatment of telomelysin and paclitaxel compared to paclitaxel alone $(\mathrm{P}<0.05)$. (C) Effects of telomelysin and cisplatin on vessel density. Statistical significance was not observed in each group $(\mathrm{P}<0.05)$. Data represent mean values $( \pm \mathrm{SD})$. Significant difference was analyzed by Student's paired t-test.

previously (26). Similarly to the other results (12), elevated levels of telomerase activity were observed in the all cell lines, but the extent of mRNA expression of hTERT was not significantly correlated with telomelysin $\mathrm{ID}_{50}$. 
The infection efficiency of the currently available adenovirus agent, which is derived from human adenovirus serotype 5, varies widely depending on expression of the coxsackievirus-adenovirus receptor (CAR) (28). Therefore, the interaction of the adenovirus with CAR on the cell surface is a key mechanism by which adenoviral agents enter cells. Some studies have shown that anticancer agents such as FR901228, etoposide and topotecan, increase adenoviral infection via enhancement of CAR expression on cancer and normal cells $(14,29,30)$. Based on these studies, we investigated the relative mRNA expression of CAR in 17 HNSCC cell lines and evaluated the correlation of CAR with telomelysin $\mathrm{ID}_{50}$. The mRNA expression of CAR was not significantly correlated with the efficacy of telomelysin, although the mRNA expression of CAR was observed in all 17 HNSCC cell lines. For the telomelysin $\mathrm{ID}_{50}$ for day 3 after the treatment, cell lines that showed relative mRNA expression of CAR that was $>0.4$ of, tended to low telomelysin $\mathrm{ID}_{50}$. A statistical correlation was not observed between mRNA expression and telomelysin $\mathrm{ID}_{50}$ for day 3 , but a relative mRNA expression of CAR appears to the efficacy of telomelysin infection and the antitumor activity at early stages after telomelysin treatment. For day 5 or 7 after telomelysin treatment, no significant correlation was observed between mRNA expression of CAR and telomelysin $\mathrm{ID}_{50}$. Therefore, another factor must be involved in the long-term efficacy of telomelysin treatment.

Next we investigated the antitumor effect of telomelysin combined with the chemotherapeutic agents paclitaxel or cisplatin. Fujiwara et al reported that combination therapy of telomelysin and docetaxel produced additive therapeutic benefits over either individual modality and that docetaxel was considered to be innocuous for replication of telomelysin because this agent did not inhibit DNA synthesis (31). Watanabe et al reported that FR901228 with or without telomelysin had no effect on cell cycle distribution and that FR901228 might be an appropriate partner for this oncolytic adenovirus because it does not affect the virus life cycle. To clarify the influence of paclitaxel and cisplatin on the antitumor effects of telomelysin, we studied combination therapy of telomelysin with paclitaxel or cisplatin in three protocols: telomelysin and chemotherapy concurrently, telomelysin then chemotherapy, chemotherapy then telomelysin. For the combination of telomelysin and paclitaxel, the all three protocols showed an additive antitumor effect in the xenograft model. Among these three protocols, concurrent administration was most effective. This result supports the idea that paclitaxel is an appropriate partner with telomelysin therapy for HNSCC and has no influence on the distribution of telomelysin in cancer cells. Regarding cisplatin, cisplatin treatment then telomelysin, but not the other protocols, showed an additive antitumor effect. Based on this result, we conclude that cisplatin will affect the negative influence on the antitumor activity of telomelysin. One reason for this result might be that cisplatin effects the cell cycle. Cisplatin arrests the cell cycle in $\mathrm{G} 1$ phase. Adenoviral E1A directs the progression of cells from $\mathrm{G} 1 / \mathrm{G} 2$ phase to $\mathrm{S}$ phase to optimize viral DNA replication (31). So, cisplatin might have deleterious effects on viral replication. Furthermore, cisplatin may affect the DNA of adenoviruses directly, because cisplatin is an anti-DNA synthesis agent.

On the other hand, paclitaxel is an anticancer agent that targets microtubules and arrests the cell cycle in G2 and M phases. These mechanisms induce apoptosis and does not inhibit DNA synthesis of host cells.

To evaluate apoptotic events of antitumor effect in vivo, the TUNEL method was utilized. Regarding telomelysin alone, few apoptotic events were observed. We previously reported that telomelysin induced apoptotic cell death in the murine SCC model (26). In the present study we used BALB/c nu/nu mice, in which the immune system does not have active $\mathrm{CD} 4^{+}$ and $\mathrm{CD} 8^{+} \mathrm{T}$ cells.

The apoptotic indexes in the combined treatment were higher compared with paclitaxel or cisplatin alone. Especially, concurrent telomelysin and paclitaxel and for telomelysin then panlitaxel showed significantly higher apoptotic indexes.

To evaluate vessel density in vivo, immunohistochemical staining was used. Vessels were detected by staining with an anti-CD31 antibody. Paclitaxel decreased the number of CD31-positive endothelial cells (32-34) and this supports the antiangiogenic activity of paclitaxel. But the combined treatment of telomelysin with cisplatin induced no antiangiogenic activity. Regarding angiogenesis, synergistic effects were not observed in either combination therapy of telomelysin and paclitaxel or cisplatin.

In conclusion, our results demonstrate that telomelysin and paclitaxel lead to a higher antitumor effect than the treatment of either agent alone on HNSCC xenografts. Therefore, paclitaxel is a promising agent for combination therapy with telomelysin. In contrast, cisplatin interfere with the replication and antitumor activity of telomelysin.

\section{References}

1. Reuter CW, Morgan MA and Eckardt A: Targeting EGF-receptorsignalling in squamous cell carcinomas of the head and neck. Br J Cancer 96: 408-416, 2007.

2. Forastiere AA, Goepfert H, Maor M, et al: Concurrent chemotherapy and radiotherapy for organ preservation in advanced laryngeal cancer. N Engl J Med 349: 2091-2098, 2003.

3. Kirn D, Martuza RL and Zwiebel J: Replication-selective virotherapy for cancer: Biological principles, risk management and future directions. Nat Med 7: 781-787, 2001.

4. Nemunaitis J, Khuri F, Ganly I, et al: Phase II trial of intratumoral administration of ONYX-015, a replication-selective adenovirus, in patients with refractory head and neck cancer. J Clin Oncol 19: 289-298, 2001.

5. Harrison D, Sauthoff H, Heitner S, Jagirdar J, Rom WN and Hay JG: Wild-type adenovirus decreases tumor xenograft growth, but despite viral persistence complete tumor responses are rarely achieved-deletion of the viral E1b-19-kD gene increases the viral oncolytic effect. Hum Gene Ther 12: 1323-1332, 2001.

6. DeWeese TL, van der Poel H, Li S, et al: A phase I trial of CV706, a replication-competent, PSA selective oncolytic adenovirus, for the treatment of locally recurrent prostate cancer following radiation therapy. Cancer Res 61: 7464-7472, 2001.

7. Reid T, Galanis E, Abbruzzese J, et al: Hepatic arterial infusion of a replication-selective oncolytic adenovirus (d11520): phase II viral, immunologic, and clinical endpoints. Cancer Res 62: 6070-6079, 2002.

8. Hamid O, Varterasian ML, Wadler S, et al: Phase II trial of intravenous CI-1042 in patients with metastatic colorectal cancer. J Clin Oncol 21: 1498-1504, 2003.

9. Blackburn EH: Structure and function of telomeres. Nature 350: 569-573, 1991 . 
10. Kim NW, Piatyszek MA, Prowse KR, et al: Specific association of human telomerase activity with immortal cells and cancer. Science 266: 2011-2015, 1994.

11. Shay JW and Wright WE: Telomerase activity in human cancer. Curr Opin Oncol 8: 66-71, 1996.

12. Umeoka T, Kawashima T, Kagawa S, et al: Visualization of intrathoracically disseminated solid tumors in mice with optical imaging by telomerase-specific amplification of a transferred green fluorescent protein gene. Cancer Res 64: 6259-6265, 2004.

13. Kawashima T, Kagawa S, Kobayashi N, et al: Telomerasespecific replication-selective virotherapy for human cancer. Clin Cancer Res 10: 285-292, 2004.

14. Watanabe T, Hioki M, Fujiwara T, et al: Histone deacetylase inhibitor FR901228 enhances the antitumor effect of telomerasespecific replication-selective adenoviral agent OBP-301 in human lung cancer cells. Exp Cell Res 312: 256-265, 2006.

15. Taki M, Kagawa S, Nishizaki M, et al: Enhanced oncolysis by a tropism-modified telomerase-specific replication-selective adenoviral agent OBP-405 ('Telomelysin-RGD'). Oncogene 24: 3130-3140, 2005.

16. Qin M, Chen S, Yu T, Escuadro B, Sharma S and Batra RK: Coxsackievirus adenovirus receptor expression predicts the efficiency of adenoviral gene transfer into non-small cell lung cancer xenografts. Clin Cancer Res 9: 4992-4999, 2003.

17. Tomko RP, Xu R and Philipson L: HCAR and MCAR: the human and mouse cellular receptors for subgroup $\mathrm{C}$ adenoviruses and group B coxsackieviruses. Proc Natl Acad Sci USA 94: 3352-3356, 1997

18. Bergelson JM, Cunningham JA, Droguett G, et al: Isolation of a common receptor for Coxsackie B viruses and adenoviruses 2 and 5. Science 275: 1320-1323, 1997.

19. Li D, Duan L, Freimuth P and O'Malley BW Jr: Variability of adenovirus receptor density influences gene transfer efficiency and therapeutic response in head and neck cancer. Clin Cancer Res 5: 4175-4181, 1999.

20. Haviv YS, Blackwell JL, Kanerva A, et al: Adenoviral gene therapy for renal cancer requires retargeting to alternative cellular receptors. Cancer Res 62: 4273-4281, 2002.

21. Li Y, Pong RC, Bergelson JM, et al: Loss of adenoviral receptor expression in human bladder cancer cells: a potential impact on the efficacy of gene therapy. Cancer Res 59: 325-330, 1999.

22. Kim M, Zinn KR, Barnett BG, et al: The therapeutic efficacy of adenoviral vectors for cancer gene therapy is limited by a low level of primary adenovirus receptors on tumour cells. Eur J Cancer 38: 1917-1926, 2002.
23. Kim JS, Lee SH, Cho YS, Kim YH and Lee JH: Ectopic expression of the coxsackievirus and adenovirus receptor increases susceptibility to adenoviral infection in the human cervical cancer cell line, SiHa. Biochem Biophys Res Commun 288: 240-244, 2001.

24. Pearson AS, Koch PE, Atkinson N, et al: Factors limiting adenovirus-mediated gene transfer into human lung and pancreatic cancer cell lines. Clin Cancer Res 5: 4208-4213, 1999.

25. Okegawa T, Li Y, Pong RC, Bergelson JM, Zhou J and Hsieh JT: The dual impact of coxsackie and adenovirus receptor expression on human prostate cancer gene therapy. Cancer Res 60: 5031-5036, 2000

26. Fujita K, Kimura M, Kondo N, et al: Anti-tumor effects of telomelysin for head and neck squamous cell carcinoma. Oncol Rep 20: 1363-1368, 2008

27. Nakayama J, Tahara H, Tahara E, et al: Telomerase activation by hTRT in human normal fibroblasts and hepatocellular carcinomas. Nat Genet 18: 65-68, 1998.

28. Wickham TJ, Mathias P, Cheresh DA and Nemerow GR: Integrins alpha $\mathrm{v}$ beta 3 and alpha $\mathrm{v}$ beta 5 promote adenovirus internalization but not virus attachment. Cell 73: 309-319, 1993.

29. Shieh GS, Shiau AL, Yo YT, et al: Low-dose etoposide enhances telomerase-dependent adenovirus-mediated cytosine deaminase gene therapy through augmentation of adenoviral infection and transgene expression in a syngeneic bladder tumor model. Cancer Res 66: 9957-9966, 2006.

30. Hemminki A, Kanerva A, Liu B, et al: Modulation of coxsackieadenovirus receptor expression for increased adenoviral transgene expression. Cancer Res 63: 847-853, 2003.

31. Fujiwara T, Kagawa S, Kishimoto H, et al: Enhanced antitumor efficacy of telomerase-selective oncolytic adenoviral agent OBP-401 with docetaxel: preclinical evaluation of chemovirotherapy. Int J Cancer 119: 432-440, 2006.

32. Fox WD, Higgins B, Maiese KM, et al: Antibody to vascular endothelial growth factor slows growth of an androgenindependent xenograft model of prostate cancer. Clin Cancer Res 8: 3226-3231, 2002.

33. Sweeney CJ, Miller KD, Sissons SE, et al: The antiangiogenic property of docetaxel is synergistic with a recombinant humanized monoclonal antibody against vascular endothelial growth factor or 2-methoxyestradiol but antagonized by endothelial growth factors. Cancer Res 61: 3369-3372, 2001.

34. Belotti D, Vergani V, Drudis T, et al: The microtubule-affecting drug paclitaxel has antiangiogenic activity. Clin Cancer Res 2: 1843-1849, 1996. 